\title{
SwarmRail: A Novel Overhead Robot System for Indoor Transport and Mobile Manipulation
}

\author{
Martin Görner ${ }^{1}$, Fabian Benedikt ${ }^{1,2}$, Ferdinand Grimmel ${ }^{1,2}$, and Thomas Hulin ${ }^{1}$
}

\begin{abstract}
SwarmRail represents a novel solution to overhead manipulation from a mobile unit that drives in an aboveground rail-structure. The concept is based on the combination of omnidirectional mobile platform and L-shaped rail profiles that form a through-going central gap. This gap makes possible mounting a robotic manipulator arm overhead at the underside of the mobile platform. Compared to existing solutions, SwarmRail enables continuous overhead manipulation while traversing rail crossings. It also can be operated in a robot swarm, as it allows for concurrent operation of a group of mobile SwarmRail units inside a single rail network. Experiments on a first functional demonstrator confirm the functional capability of the concept. Potential fields of applications reach from industry over logistics to vertical farming.
\end{abstract}

\section{INTRODUCTION}

According to reports of leading research institutes and consulting companies, the factory of the future will be characterized by a highly flexible, modular, dynamically reconfigurable and multidirectional layout of the plant structure as well as an intensive use of digital technologies such as smart and collaborative robots [1], [2]. The focus shifts from fixed production lines to modular conveyors or mobile robotic carrier units like the robot presented in [3] that move workpieces or component parts to reconfigurable robotic workcells [4], [5], as also illustrated in DLR's vision of the factory of the future in Fig. 1. Mainly relying on groundbased systems, most of the proposed concepts will lead to densely used shop floors, while the space from above is only occupied by infrastructure such as lighting, power supply or simple part feeding lines. However, accessing the workspace from above would tremendously increase flexibility and adaptability of a production process.

To date, there is no system known to the authors that can provide such access from above for a higher number of mobile robotic units across the whole area of the shop floor. Traditional omnidirectional mobile platforms [7], [8], [9], [10], [11] operate on the ground. Gantry robots or linear axes are usually limited to a local environment while only supporting a limited number of manipulators as for example DLR's advanced multifunctional cell for aerospace carbonfiber-reinforced plastics production [12]. Robotic solutions for automated warehouses, such as the Ocado or AutoStore logistic systems [13], are not able to drive while manipulating or lifting goods.

\footnotetext{
${ }^{1}$ All authors are with the Institute of Robotics and Mechatronics, German Aerospace Center (DLR), Wessling, Germany thomas.hulin@dlr.de

${ }^{2}$ Fabian Benedikt and Ferdinand Grimmel are additionally with the Munich University of Applied Sciences, Munich, Germany
}

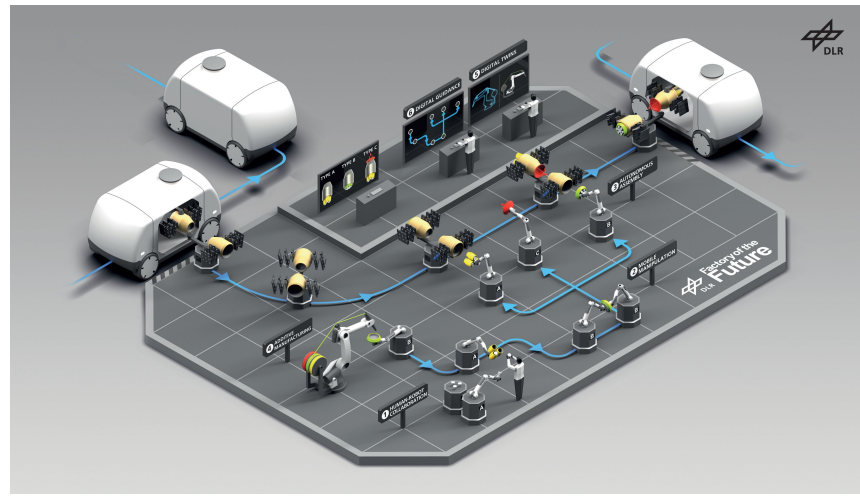

Fig. 1. Rendering of DLR's vision of the Factory of the Future [6]

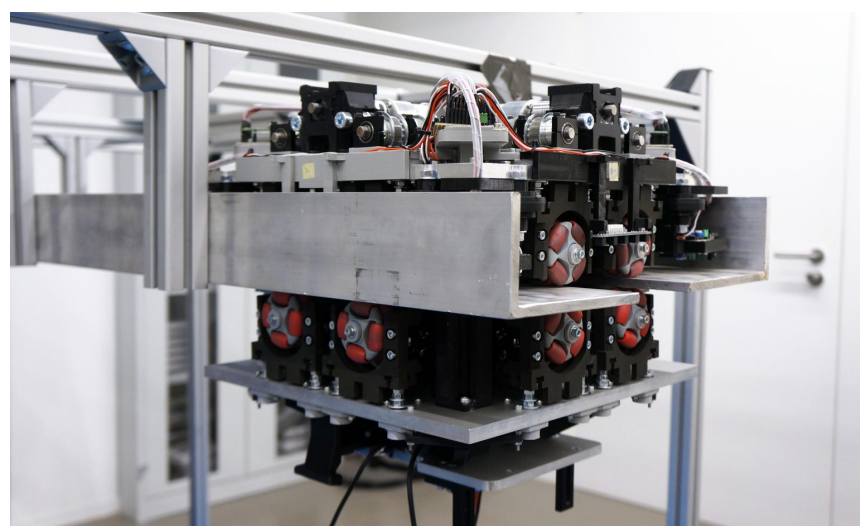

Fig. 2. SwarmRail demonstrator: Mobile unit and top-mounted rail

In comparison, the SwarmRail system aims at providing transport and mobile manipulation from above across the whole shop floor. The system consists of omnidirectionally mobile robotic units as shown in Fig. 2 and a special grid of rails with passive intersections. This beneficial combination primarily allows for

- continuous overhead manipulation while driving and even while traversing rail crossings, and

- concurrent operation of a group of mobile units inside a single rail network.

This article presents in detail the SwarmRail mobility concept - i.e. the design of the mobile robotic unit and the specific properties of the rail system - and discusses its potential. Sec. II introduces the overall conceptual idea of the SwarmRail system. Next, Sec. III explains the design of rail crossings and the development of a rapid-prototyping-based mobile robotic unit. Finally, Sec. IV presents an experimental validation of the mobility concept before Sec. V concludes with a brief summary and an outlook on future developments. 


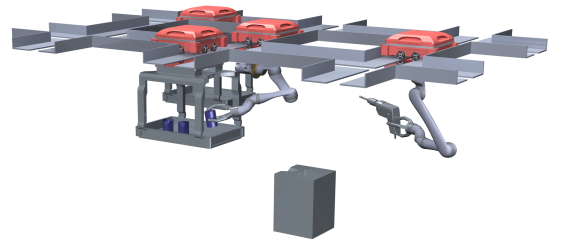

(a) Part delivery

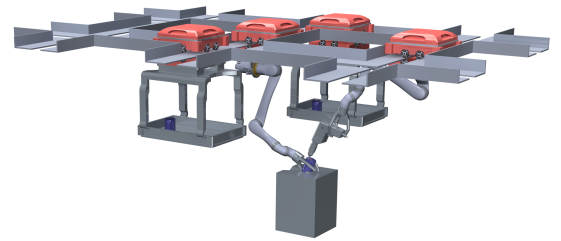

(b) Part processing

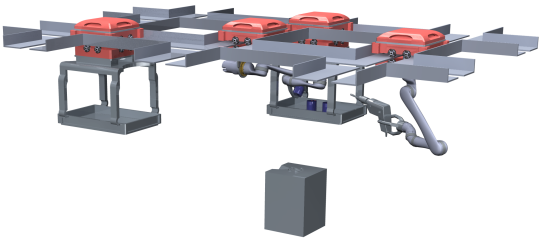

(c) Part finished

Fig. 3. Rendering of an exemplary production process sequence using the SwarmRail system

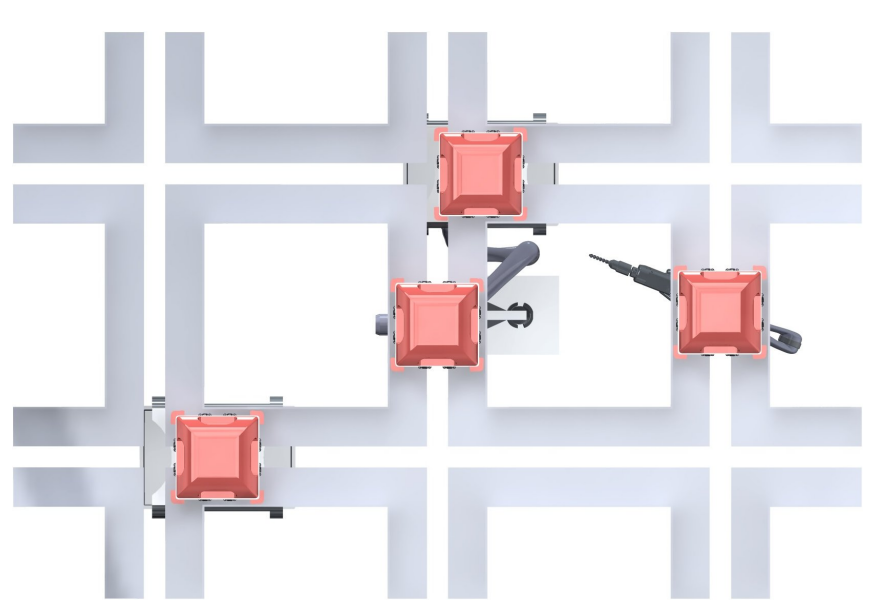

(a) Top view
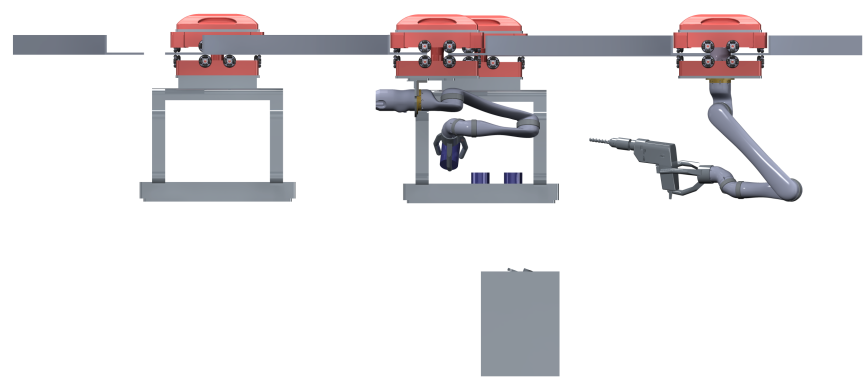

(b) Side view

Fig. 4. Rendering of different views of the SwarmRail system

\section{Conceptual IdeA}

As already outlined, the main conceptual idea of the SwarmRail system is to provide access to the factory workspace from above, and thus to add flexibility to the production process. Consequently, tasks such as transport or robotic manipulation are not limited to ground-based systems anymore, but can be performed by or in cooperation with an overhead robotic unit. To achieve a broad coverage across the shop floor with multiple mobile robotic units, the SwarmRail system employs a special grid of rails as shown in Fig. 4(a). Those rails are characterized by tracks that do not intersect at a crossing but form an intersecting gap. Within this unique setup, each mobile unit of the SwarmRail system consists of two parts that are connected through the gap - an upper part that runs on top of the tracks and supports the weight and a lower part that holds a transport box or a manipulator as depicted in Fig. 4(b). The use of special structural connectors allows to mount the rail grid from above to the ceiling or
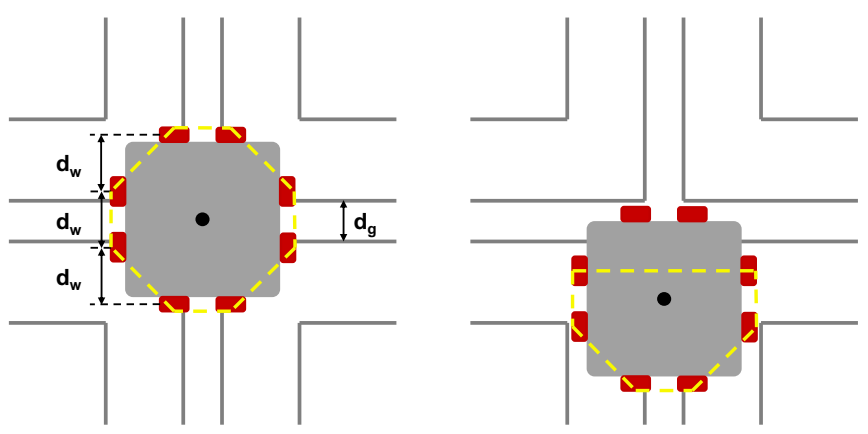

Fig. 5. Kinematic layout of an omni-wheel-based mobile unit and its support polygon (yellow) in two different contact situations

an intermediate level structure. By this, the space below the rail grid is kept free of structural parts that could interfere with the workspace of a suspended manipulator.

In order to cross the gap at an intersection, each mobile unit requires at least four sets of wheels as shown in Fig. 5. Those have to be arranged such that the wheels in contact always form a stable polygon of support. In case of the SwarmRail system, omni-wheels are the optimal choice since they allow for omnidirectional mobility with a minimum number of actuators. Thus, a setup with eight omni-wheels enables the mobile unit to safely cross the gap and to move into any possible direction at an intersection. Within this setup, it is important that the omni-wheels are placed at an equal distance $d_{w}$ that is larger than the gap width $d_{g}$. A more detailed discussion of the kinematic layout of the mobile unit follows in Sec. III-B

One of the major advantages of the SwarmRail system is the simple structure of the rail grid that allows the mobile units to change the direction at an intersection without the need of track switches or other active parts. Therefore, many mobile units can operate in parallel and can reach their goal locations along a variety of different paths, which adds flexibility to the overall production process. A simple example for such process involving four mobile units is given in Fig. 3. Herein, one mobile unit delivers a part that is put into a fixture by a manipulator mounted to a second mobile unit. A third mobile unit holds another manipulator that processes the part while a fourth unit carries the processed part to the next production step. 


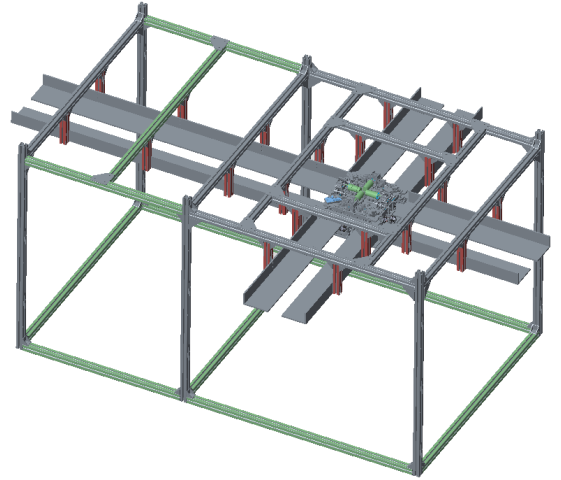

(a)

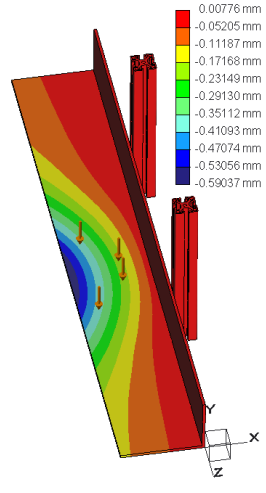

(b)
Fig. 6. a) Rendering of the built rail structure with one crossing and b) FEM rail stress analysis of an L-profile with dimensions $150 \times 75 \mathrm{~mm}$, a thickness of $8 \mathrm{~mm}$, and a total load of $4 \times 50 \mathrm{~N}$ result in a maximum vertical deflection of $0.595 \mathrm{~mm}$.

\section{Demonstrator}

In order to evaluate the core functional capabilities of the SwarmRail system, a rapid-prototyping-based demonstrator has been built within the scope of a student project [14], [15]. This demonstrator comprises a single mobile unit and a single rail crossing at a total cost of less than $5000 €$. The following lines describe its technical details.

\section{A. Rail System}

While the future rail grid will be built of quadratic, rectangular or hexagonal elements that are connected to form a gap as shown in the supplementary video, the SwarmRail demonstrator only comprises a single rail intersection with three $1 \mathrm{~m}$ branches and one $2 \mathrm{~m}$ branch (see Fig. 6(a)). This setup is sufficient to validate the mobility concept and the basic control algorithm. The tracks of the demonstrator are built from standard off-the-shelf L-shaped aluminum profiles that provide a single track width of $150 \mathrm{~mm}$ and a height of $75 \mathrm{~mm}$ for mounting. The gap was chosen to be $80 \mathrm{~mm}$ which results in a total width of $380 \mathrm{~mm}$ for each rail branch. The complete rail intersection is mounted to a supporting frame such that the whole space below the rails is free of structural parts as shown above in Fig. 2. The load capacity of the $8 \mathrm{~mm}$ thick aluminum profiles has been evaluated using FEM simulations and is sufficient to hold a mobile unit of $20 \mathrm{~kg}$ with negligible deformations. Fig. $6(\mathrm{~b})$ shows the FEM results obtained for a worst-case assumption in which each omni-wheel applies a vertical force of $50 \mathrm{~N}$ (marked by the four orange arrows) resulting in a maximum total deflection of $0.59 \mathrm{~mm}$. This case only considers a single track, which translates to a supported mass of about $40 \mathrm{~kg}$ for a normal rail consisting of two tracks.

\section{B. Mobile Unit}

The mobile unit of the SwarmRail demonstrator consists of an upper and a lower part that are connected through the gap. The mobile unit would already work with the upper part only. However, to support robotic manipulation which may cause high reaction torques, a lower part was added that avoids

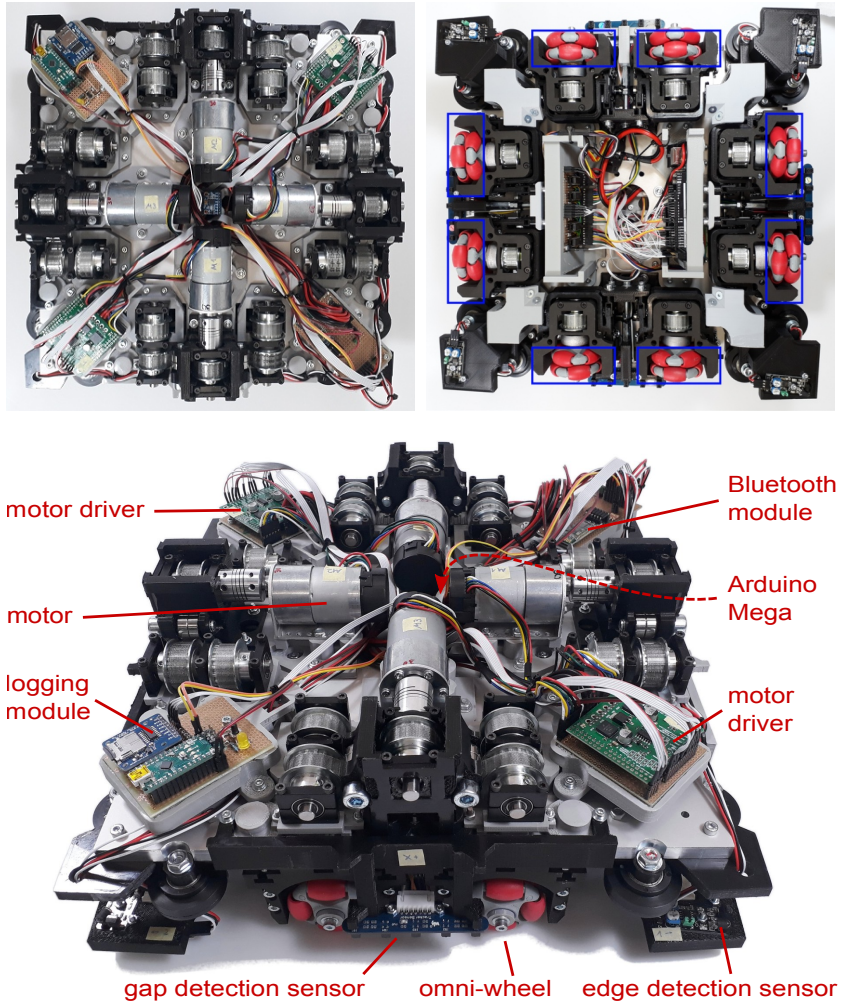

Fig. 7. Upper part of mobile unit

TABLE I

SYSTEM SPECIFICATIONS OF SWARMRAIL UNITS.

\begin{tabular}{ll}
\hline \multicolumn{1}{c}{ system specifications } \\
\hline size & $362 \mathrm{~mm} \times 362 \mathrm{~mm} \times 263 \mathrm{~mm}(1 \times \mathrm{w} \times \mathrm{h})$ \\
mass & approx. 17.5 kg \\
max. speed & $0.5 \mathrm{~m} / \mathrm{s}$ \\
payload & $10 \mathrm{~kg}$ \\
motors & $4 \times$ brushed DC gearmotor (Pololu 4752) \\
sensors & $4 \times$ IR gap detection (EXP-R63-145) \\
& $8 \times$ IR edge detection (Iduino ST1081) \\
& $1 \times$ IMU (6DOF MPU6050) \\
wheels & $8 \times$ driven omni-wheels (Rotacaster R2-0504-60) \\
& $8 \times$ passive omni-wheels on lower part \\
CPU & Arduino Mega 2560 \\
control freq. & $100 \mathrm{~Hz}$ \\
\hline
\end{tabular}

tilting. The upper part is shown in Fig. 7 and hosts eight omni-wheels, all drives, sensors, and electronics and provides active mobility. The lower part has the same kinematic setup regarding the wheels but is completely passive. It contains a mounting point for a manipulator or a transport box.

The following paragraphs provide details on mechanical aspects, the electronics and the controller.

1) Mechanical Design: Starting from the requirements imposed by the rails, a kinematic layout with eight omniwheels as depicted in Fig. 5 was chosen. The advantage of this layout is that it achieves omnidirectional mobility with only four actuators, saving cost and space. In comparison, the same configuration but with Mecanum wheels would require eight motors; one for each wheel. Since its configuration is statically overdetermined, the mobile unit requires a suspen- 


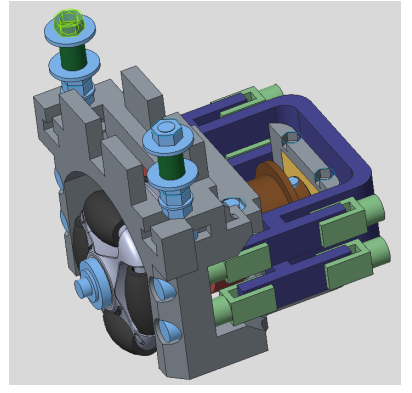

(a)

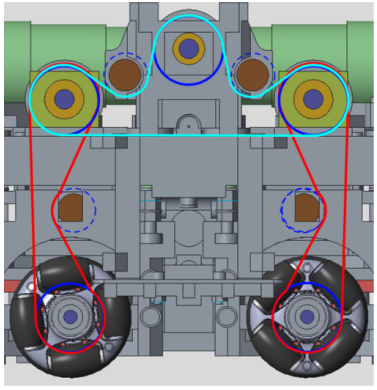

(b)
Fig. 8. a) Single wheel suspension and b) tooth belt transmission of the SwarmRail mobile unit

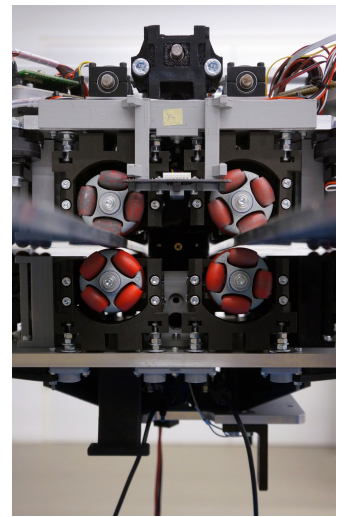

(a)

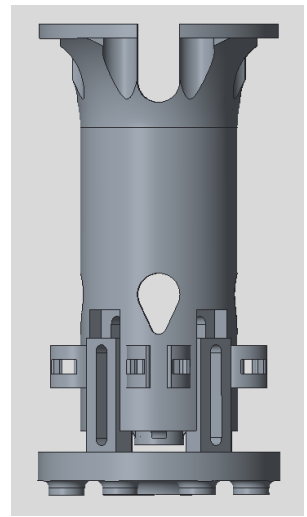

(b)
Fig. 9. Mechanical connector between upper and lower part of the mobile unit through the rail gap a) on the real demonstrator and b) in the CAD model

sion system to enforce the contact in between the wheels and the tracks. This is solved using a spring-loaded single wheel suspension with a parallel bar linkage as shown in Fig. 8(a). This mechanism allows each omni-wheel to move up and down independently while keeping it vertical. The reason for this compact solution is twofold. First, omni-wheels are highly sensitive to tilt, and second, the central space of the mobile unit is reserved for the structural connection of the upper and the lower part. Furthermore, a single wheel suspension is a good fit with the actuation concept that aims at driving both wheels on one side with a single motor. This motor is connected to the wheels by a spring-loaded toothbelt transmission as shown in Fig. 8(b). Being spring-loaded, it easily adjusts to the up and down motions of the single wheel suspension. A critical parameter is the motion range of the suspension. This range needs to be high enough to cope with the small height variations of the tracks but small enough to avoid severe collisions of the wheel with the track edge while crossing the gap in an extended state. For the demonstrator, this motion range is currently set to $2 \mathrm{~mm}$. The connecting part shown in Fig. 9 is manually adjustable in its length to preset the contact forces in between the wheels and the tracks. The integrated mechanism would further allow a future actuation of the connecting part to enable contact force control.

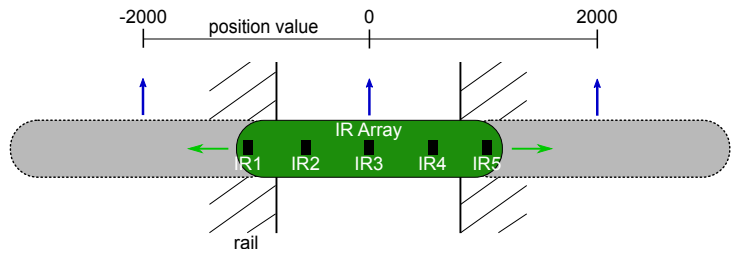

Fig. 10. IR sensor array (EXP-R63-145) above rail gap. The implemented algorithm results in values between -2000 and 2000 for left and right position offsets respectively.

2) Electronics: One role of the electronics is to sense and process data necessary for driving the mobile unit centrally in the rail and over crossings. To this end, the unit is equipped with two different types of sensors for detecting rail gaps and crossings respectively (see Fig. 10). IR arrays are used to determine the position of the mobile unit relative to the rail gaps. These gap detection sensors are a simple and costeffective variant of sensors and are frequently employed for line tracking tasks of mobile robots. For optimal positioning of the mobile SwarmRail unit in each direction, four of these IR sensor arrays are integrated - one on each side of the robot (see Fig. 7).

In principle, these sensors can also be used to detect rail crossings. However, to enable fast driving speeds, crossings need to be detected well before reaching their center such that there is enough time for decelerating the mobile unit prior to turning. Hence, the unit is equipped with another eight IR sensors (Iduino ST1081), two in each corner of the mobile unit. These IR sensors detect rail crossings by measuring the reflection at the walls of the L-shaped rail profiles. This arrangement is well suited for a driving speed of more than $0.5 \mathrm{~m} / \mathrm{s}$.

The four integrated geared motors of type 37Dx68L are DC motors with a rotary encoder that can be used to determine the position of the shaft. Their maximum rotation speed is $350 \mathrm{rpm}$ with which theoretically a maximum driving speed of $0.88 \mathrm{~m} / \mathrm{s}$ can be achieved with the selected wheels. This first demonstrator is currently powered by an external power supply via a cable but could also use rechargeable batteries, which is the desired solution for the SwarmRail system. The electronics also comprise a Bluetooth communication module that enables the mobile unit being controlled from a smartphone and an Arduino Mega which runs the control algorithm that is described in the following lines.

3) Control: The controller operates the mobile unit inside the rail structure. It processes user-commands in a state machine and it switches between the states of accelerating, driving along rails, approaching rail crossing, centering inside rail crossings, rotating, and remaining stationary (see also Fig. 11).

During driving, the mobile unit centers itself with regard to the rail gap to avoid contact between the mechanical structure of the unit and the rails. Fig. 12 shows two basic correction concepts for this task. One concept is traditional skid steering (Fig. 12(a)), while the other one employs the omni-wheels that are oriented perpendicular to the principal direction 


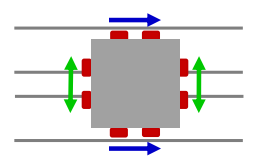

1

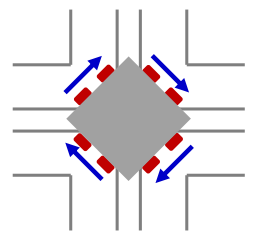

4
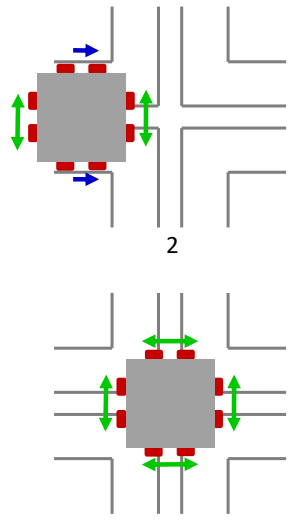
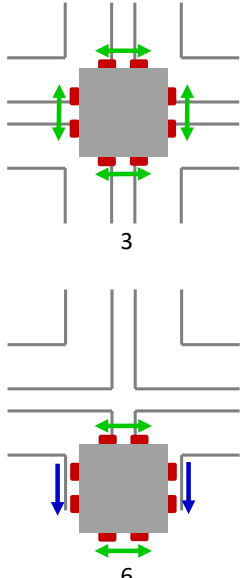

Fig. 11. Different states for driving through a rail crossing with a $90^{\circ}$ change of orientation. The blue arrows indicate the principle motion direction, while the green arrows represent the corrective direction for centering the unit with regard to the gap.
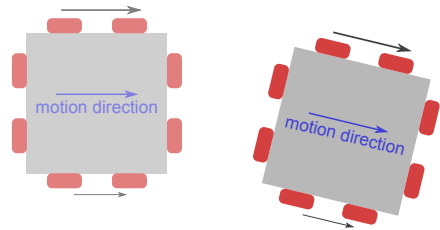

(a)

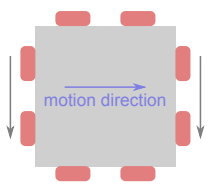

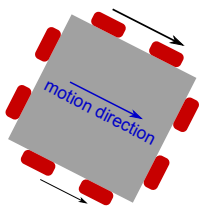

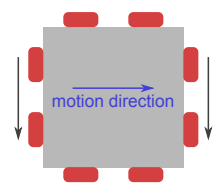

(b)

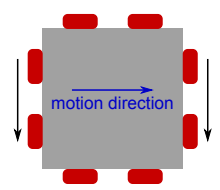

Fig. 12. Two different possible control concepts for centering the mobile unit above a rail gap. The blue arrow indicates the principal direction of movement. The black arrows illustrate the corrective action of the centering concepts. The second concept is used for driving inside the rail profiles since it maintains the orientation of the unit.

of movement (Fig. 12(b)). In contrast to skid steering that results in a curved corrective motion, the second concept directly influences the orientation or lateral position of the mobile unit by superposition of motions. Hence, it is used for driving along the rails.

By using this concept, the task of a wheel is either to move (rotate or translate) the unit, or to correct its position above the rail gap. In detail, each pair of wheels orthogonal to the driving direction uses the position signal of the corresponding IR sensor array to apply an individual corrective motion in response to a detected offset. Inside a crossing, the mobile unit is centered by a similar approach using all four sensor arrays and wheel pairs.

For centering, a classical PID-controller runs on the mi-
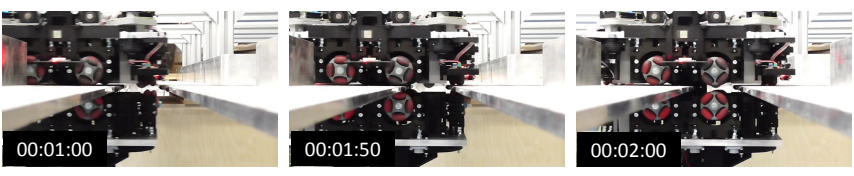

(a)
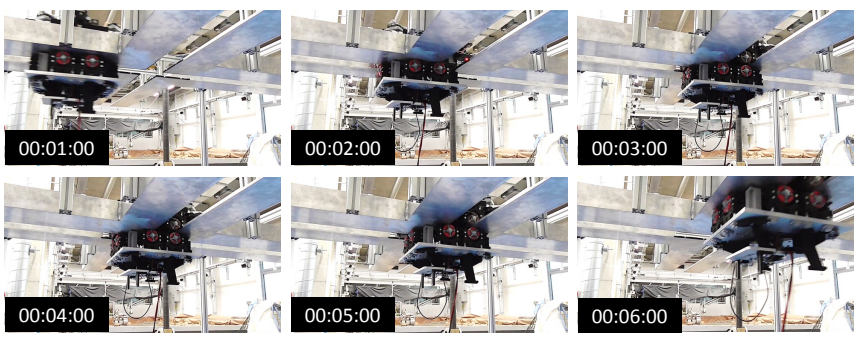

(b)
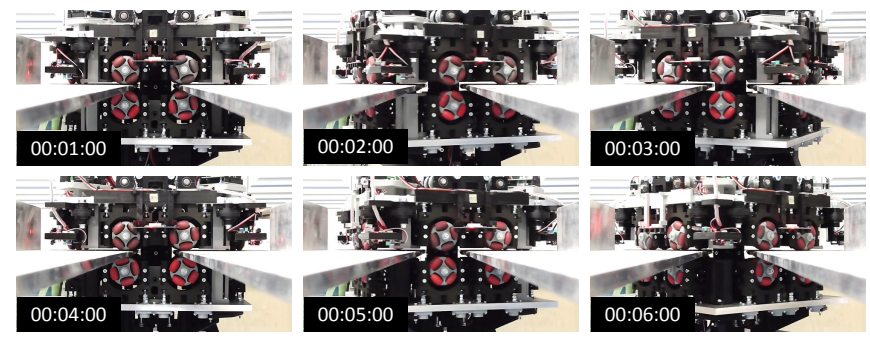

(c)

Fig. 13. Photo sequences. a) mobile unit enters a crossing, b) mobile unit traverses a rail crossing and changes its principal direction of motion without changing its orientation, c) mobile unit turns on the spot at the center of a rail crossing

crocontroller that controls the wheel rotation velocity. This controller employs an update rate of $100 \mathrm{~Hz}$ and is tuned according to the Ziegler-Nichols oscillation method [16].

\section{EXPERIMENTAL VALIDATION}

To validate the mobility concept of the SwarmRail system, several experiments were performed using the demonstrator. First of all, the omnidirectional mobility of the upper part of the mobile unit was tested on plain floor. For this purpose, the unit was driven in each direction by commanding a feedforward velocity to opposing wheel pairs. Being placed on a line, the accuracy of the velocity control of each wheel pair could be tested. The result was very convincing since the robot only showed very small lateral deviations of less than $5 \%$ from the line. This indicates that the suspension system ensures sufficient traction of all wheels and that all four velocity controllers work equally well. Next, turning in both directions was tested. Again, only very small superimposed translations of less than $5 \mathrm{~cm}$ were observed for a rotation of $360^{\circ}$. Combining both motions into a curved path further proved full omnidirectional mobility of the SwarmRail unit.

Following these mobility tests, the mobile unit was set into the rails. First, the centering controller was tested within the crossing by displacing the unit manually from the center. The initial controller gains were adjusted by increasing the proportional gain to achieve an accuracy of a few millimeters. Next, the driving and the centering controller were tested while driving along the $2 \mathrm{~m}$ branch of the test setup. The 
mobile unit achieved speeds of up to $0.5 \mathrm{~m} / \mathrm{s}$ while being properly centered above the gap. The final tests involved driving through a crossing and turning. Initially, some of the wheels lost traction while passing the crossing such that the mobile unit did not properly enter the opposite branch. The critical phase was when the leading pair of wheels passed the orthogonal gap (see Fig. 13(a)). In this state, the mobile unit could initially not properly center above the gap since only the trailing pair of wheels was left for control. However, several adjustments solved this problem. First of all, the driving speed within an intersection was limited to $0.3 \mathrm{~m} / \mathrm{s}$. Additionally, small round plastic caps were mounted to the rail edges forming the gap to allow for a smoother transition of the wheels from the gap onto the rail. Next, the rail surface was slightly roughened to improve traction. As a result, the SwarmRail unit is now able to smoothly transition the crossing in all four directions. Fig. 13(b) shows a sequence of video images wherein the mobile unit enters a crossing from the left, stops, centers itself, and moves towards the observer.

The last test performed within the crossing was plain turning on the spot in multiples of $90^{\circ}$. While this motion is not necessary for properly moving through an orthogonal grid of rails, it is required for passing a crossing within a hexagonal structure. Furthermore, turning on the spot allows to reorient a mounted manipulator that has a nonsymmetric workspace with respect to the vertical axis of the SwarmRail unit. The sequence in Fig. 13(c) exemplarily shows a turning motion from below.

So far, the load capacity has not been evaluated in detail but initial experiments show that an increased weight even improves traction. The mechanically critical parts are the wheels and the structure connecting the upper and the lower platform. However, in their current state they are able to support more than the $17.5 \mathrm{~kg}$ mass of the mobile unit and the desired additional payload of $10 \mathrm{~kg}$. Detailed locomotion tests with symmetrically and asymmetrically suspended payloads as expected for a mounted manipulator are the subject of ongoing work.

All of the elementary motions of the mobile robotic unit, such as driving straight forward, passing a crossing in different directions, and turning on the spot, are demonstrated in the supplementary video.

\section{CONCLUSION}

This article introduced SwarmRail - a novel conceptual robotic system for overhead transport and manipulation. Especially in conjunction with ground-based mobile robots, SwarmRail will enable completely new and highly flexible production processes within the reconfigurable factory of the future. It will allow production process designers to assign manufacturing steps and transport tasks either to a ground-based mobile robot or a top-mounted SwarmRail unit. Furthermore, tasks could be assigned to heterogeneous groups of robots that dynamically form small productions cells at changing locations of the plant.
The SwarmRail system itself shows many beneficial features as listed below. These features are not only useful in industrial applications, but are also relevant to other fields such as logistics [17] or vertical farming [18].

F1 The available workspace is exploited from above

F2 Many mobile units can be used simultaneously

F3 The units can be equipped with different payloads

F4 The units can work jointly

F5 Using the same rail structure, the units can differ in cost and performance

F6 The system is highly redundant

F7 Continuous manipulation while driving is possible

F8 The rail structure is passive, easily extendable and allows for various topologies

F9 The units can rotate in crossings

F10 The units support high interaction forces and torques of a mounted manipulator

As a first step in the development process, the mobility concept of the SwarmRail system was successfully validated by experiments using a rapid-prototyping-based demonstrator that was built within the scope of a student project. Several enabling solutions have been found and investigated with respect to the mechanism design itself as well as the basic motion control approach. Especially, the suspension system works well in conjunction with the omni-wheels and the minimal configuration with four driving motors. The performance of the intuitive motion controller with its separation of centering and propulsion tasks is very convincing. The turning capability of the mobile unit, enables the future use of very different rail grids ranging from quadratic and rectangular layouts to hexagonal grids or even a mixture thereof. This allows to create work areas with dense rail grids - i.e. intersections every two to three meters - and longer transfer routes without frequent intersections that allow for higher velocities.

The encouraging results obtained with the present demonstrator are the starting point for a high performance followup system. This system will involve a larger modular rail grid and multiple mobile units with manipulators mounted that will exhibit the majority of the beneficial features listed above. It will allow to develop and demonstrate increasingly complex applications involving manipulation tasks for single units that require whole body controllers and visual servoing algorithms. Furthermore, it will enable research on navigation, collision avoidance and motion planning in a real multirobot SwarmRail system.

\section{ACKNOWLEDGMENT}

The authors would like to thank their colleagues Benedikt Pleintinger, Ralph Bayer, Florian Loeffl and Harald Wagner for their contributions to the hardware demonstrator. We also thank Tilo Wüsthoff for his illustration of DLR's factory of the future vision and Roman Weitschat for the valuable discussions. 


\section{REFERENCES}

[1] D. Küpper, K. Kuhlmann, S. Köcher, T. Dauner, and P. Burggräf, The Factory of the Future. Boston Consulting Group, Dec. 2016.

[2] "Factories of the future: Multi-annual roadmap for the contractual ppp under horizon 2020," 2013, European Factories of the Future Research Association.

[3] A. Dömel, S. Kriegel, M. Kaßecker, M. Brucker, T. Bodenmüller, and M. Suppa, "Towards fully autonomous mobile manipulation for industrial environments," Int. Journal of Advanced Robotic Systems, pp. 1-19, July 2017.

[4] M. Shneier and R. Bostelman, Literature review of mobile robots for manufacturing. US Department of Commerce, National Institute of Standards and Technology, May 2015.

[5] T. Gaspar, B. Ridge, R. Bevec, M. Bem, I. Kovač, A. Ude, and Ž. Gosar, "Rapid hardware and software reconfiguration in a robotic workcell," in IEEE Int. Conf. on Advanced Robotics, July 2017, pp. 229-236.

[6] "Factory of the future: Intelligent robots for digitally-driven production," [Online]. Available: https://factory-of-the-future.dlr.de/, (Date last accessed 06. Sep. 2019).

[7] M. Wada and S. Mori, "Holonomic and omnidirectional vehicle with conventional tires," in IEEE Int. Conf. on Robotics and Automation (ICRA), vol. 4. IEEE, 1996, pp. 3671-3676.

[8] C. Ren and S. Ma, "Dynamic modeling and analysis of an omnidirectional mobile robot," in IEEE/RSJ Int. Conf. on Intelligent Robots and Systems (IROS). IEEE, 2013, pp. 4860-4865.

[9] L. Huang, Y. Lim, D. Li, and C. Teoh, "Design and analysis of a four-wheel omnidirectional mobile robot," in Int. Conf. on Autonomous Robots and Agents, 2004, pp. 425-428.

[10] M. Komori, K. Matsuda, T. Terakawa, F. Takeoka, H. Nishihara, and $\mathrm{H}$. Ohashi, "Active omni wheel capable of active motion in arbitrary direction and omnidirectional vehicle," Journal of Advanced Mechanical Design, Systems, and Manufacturing, vol. 10, no. 6, 2016.

[11] Y. Tian, S. Zhang, J. Liu, F. Chen, L. Li, and B. Xia, "Research on a new omnidirectional mobile platform with heavy loading and flexible motion," Advances in Mechanical Engineering, vol. 9, no. 9, pp. 1-15, 2017.

[12] F. Krebs, L. Larsen, G. Braun, and W. Dudenhausen, "Design of a multifunctional cell for aerospace cfrp production," The Int. Journal of Advanced Manufacturing Technology, vol. 85, no. 1-4, pp. 17-24, July 2016.

[13] "Swisslog autostore: An in-depth review of automated split case picking technology for distribution centers," [Online]. Available: http://www.mwpvl.com/html/swisslog_autostore_review.html, (Date last accessed 09. Sep. 2019).

[14] F. Benedikt, "Conceptual design and development of a mobile robotic unit for the SwarmRail-system," bachelor thesis, Munich University of Applied Sciences, Munich, Germany, Feb. 2019.

[15] F. Grimmel, "Programming a microcontroller to control mobile robotic units for the SwarmRail-system," bachelor thesis, Munich University of Applied Sciences, Munich, Germany, Apr. 2009.

[16] J. G. Ziegler and N. B. Nichols, "Optimum settings for automatic controllers," Transactions of ASME, vol. 64, pp. 759-768, Nov. 1942.

[17] M. A. Roa, D. Berenson, and W. Huang, "Mobile manipulation: Toward smart manufacturing [tc spotlight]," IEEE Robotics and Automation Magazine, vol. 22, no. 4, pp. 14-15, 2015.

[18] F. Kalantari, O. Mohd Tahir, A. Mahmoudi Lahijani, and S. Kalantari, "A review of vertical farming technology: A guide for implementation of building integrated agriculture in cities," in Advanced Engineering Forum, vol. 24. Trans Tech Publications Ltd, 2017, pp. 76-91. 